\title{
Proponer la propia pedagogía: reto sugestivo para los maestros
}

\author{
Proposing own pedagogy: a suggestive challenge to teachers \\ Propor a própria pedagogia: desafio sugestivo para \\ professores
}

\author{
Carlos Germán Juliao Vargas \\ Magíster en Estudios Sociales, Políticos y Económicos \\ Corporación Universitaria Minuto de Dios \\ cgjuliao@gmail.com
}

\section{Resumen}

Se pretende responder a la cuestión: ¿qué se entiende por pedagogía y quién es pedagogo/a? desde la perspectiva de que todo maestro genera una "teoría practica" como fruto de su quehacer educativo, pero que definir ese saber transferible que solo los maestros producen requiere un ejercicio reflexivo y crítico que no siempre sabemos realizar. Se insta a que todo maestro desentrañe su teoría implícita, para revelarla, objetivarla, comprenderla, transformarla y transferirla si es el caso.

Palabras claves: Pedagogía, educación, maestro, teoría práctica.

\begin{abstract}
It is intended to answer the question: what is understood by pedagogy and who is a pedagogue? from the perspective that every teacher generates a "practical theory" because of his educational work, but that defining that transferable knowledge that only teachers produce requires a reflective and critical exercise that we do not always know how to perform. Every teacher is urged to unravel his implicit theory, to reveal it, objectify it, understand it, transform it, and transfer it if it is the case.
\end{abstract}

Keywords: Pedagogy, education, teacher, practical theory. 


\section{Resumo}

Pretende-se responder à questão: o que se entende por pedagogia e quem é pedagogo/a? Na perspectiva de que todo professor gera uma "teoria prática" como resultado de seu trabalho educativo, mas que definir aquele saber transferível que só o professor produz, exige um exercício reflexivo e crítico que nem sempre sabemos fazer. Todo professor é instado a desvendar sua teoria implícita, a revelá-la, objetivá-la, compreendê-la, transformá-la e transferi-la, se for o caso.

Palavras-chave: Pedagogia, educação, professor, teoria prática.

\section{Introducción}

La educación es una acción/interacción social presente en todas las actividades de la vida personal y colectiva de las personas. También es un término ambiguo, y por eso no conviene partir de la pregunta ¿qué es la educación? sino de esta otra: ¿cómo usamos la palabra "educación"? Así se pueden decir muchas cosas sobre ella: que denota hechos sociales e interacciones (maestros enseñando y aprendices, proceso interactivoformativo, instituciones escolares, currículum, dispositivos didácticos, edificios, plataformas tecnológicas, normatividad jurídico-administrativa, etc.).

Así pues, la educación es una realidad histórico-social que supone una disciplina que estudie dichos hechos. Para nosotros, esa es la pedagogía. Todas las culturas la han valorado, según sus contextos, a lo largo de la historia. Para el mundo actual adquiere un sentido sin precedentes, al caracterizarnos como sociedades del saber y la información, y al descubrir su importancia en el devenir cotidiano de personas, organizaciones y colectivos. Pero ¿quién puede investigar y generar conocimiento en educación? El pedagogo/a.

Ahora bien, ¿qué se entiende por pedagogía y quién es pedagogo/a? Probablemente pocos pueden responder con seguridad dado el carácter polisémico del concepto. ¿Cómo defender hoy nuestra propia pedagogía (y hay que hacerlo) sin preguntarnos primero: ¿qué es la pedagogía? Hay poca claridad sobre este concepto, pues se define sin distinguirla de la educación.

Etimológicamente, la palabra pedagogía deriva del griego paidos (que significa niño) y agein (que significa guiar). Por ende, podemos decir que pedagogo es quien se encarga de guiar e instruir a los niños. Pero aquí surge el primer problema: ¿ello no es también para los adultos? Al 
responder afirmativamente esta duda se creó otro término (andragogo/andragogía). Usamos el concepto pedagogía a veces de forma errada $y$, por ende, su definición aún no es clara.

Por otra parte, el nacimiento de la pedagogía como disciplina ocurre a finales del siglo XVIII. Su cientificidad no era cuestionada, pero algunos la consideraban articulada con la filosofía, mientras otros la asumían como sinónimo de educación. Dependiendo del autor, la pedagogía era definida como ciencia, arte, saber o disciplina. En lo que sí había acuerdo era en su objeto: la educación.

¿Dónde está, entonces, el problema? Se debe a que toda "disciplina" responde a la idea de ciencia dominante (normativa y prescriptiva), exigiendo una contrastación empírica que en educación no se logra del todo, pues tiene que contemplar la particularidad de cada cultura, sociedad o historia, e incluso de cada sujeto.

A la pedagogía se la asoció con el saber-hacer, es decir, se consideró como una disciplina práctica. Por consiguiente, algunos pensaron que no era del todo una ciencia. Poco a poco la pedagogía se fue desacreditando y hoy en día los estudios eurocéntricos y la tradición estadounidense la consideran como una ciencia no experimental.
Continuando con la historia hallamos tres posturas, que dependen de la escuela a la que se adscriben. Según De los Ríos (2011), son las siguientes:

a. Tradición alemana: La pedagogía es la ciencia de la educación que nace con los textos escolares didácticos de Comenio y Ranke. Se la entendió desde la distinción de Dilthey entre ciencias del espíritu y naturales. Se consolida como disciplina científica correlacionando la pedagogía humanista, las ciencias empíricas de la educación y la ciencia crítica educativa.

b. Tradición francesa: El concepto de ciencias de la educación surge de la República del siglo XVIII, derivada de la Revolución francesa. Se consolida más tarde, a principios del siglo $\mathrm{XX}$, basada en un modelo de observación y cuantificación científica del "hecho educativo". Dado que se trata de un conjunto de disciplinas, con un mismo objeto de estudio (educación), la pedagogía se convierte en herramienta operativa de las ciencias de la educación.

c. Tradición anglosajonaestadounidense: Como teoría de la educación se origina en las teorías curriculares, focalizándose en lo operativo y metodológico. La tradición anglosajona une, bajo el nombre de educación, la práctica y la disciplina que la estudia, concibiendo la necesidad de un 
discurso teórico y de una apuesta práctica.

La pedagogía emerge, entonces, como una meta-disciplina educativa o un super-saber social (Flores, 1994), que reconstruye los sentidos producidos desde la perspectiva de formación del ser humano en un horizonte histórico-cultural determinado.

\section{¿Qué es, entonces, esta pedago- gía que no se deja definir?}

Fabre (2002) nos indica que la pedagogía hoy se declina en tres sentidos: (a) puede ser una teoría práctica de la educación, término prestado a Durkheim para designar una reflexión única sobre la acción educativa con fines de mejorarla; (b) esta reflexión educativa se puede formalizar en una doctrina (por ejemplo, las pedagogías de Freinet o Montessori), que surge del enfoque anterior al ser sistematizado; (c) por extensión, también puede ser, en el lenguaje cotidiano, el arte de educar o enseñar de cada maestro (cuando decimos de alguien que es "buen pedagogo").

Propongo, en este texto, ocuparnos del primer sentido (el más interesante ya que los otros dos se desprenden de él) para identificar en qué se relaciona lo que podemos hacer como maestros, visto como un desarrollo de teorías prácticas. Y asumo, de entrada, la descripción que hice de ella en mi obra Una pedagogía praxeológica (2014):
Lo que podemos señalar para nuestros propósitos, y en lo que parecen estar de acuerdo la mayoría de los teóricos es que la pedagogía es un saber del maestro acerca de su práctica o quehacer educativo que, por una parte, intenta orientarla $y$, por otra, pretende reflexionar y aprender desde y sobre ella. Se trataría, entonces, de un cuerpo de conocimientos de diversas procedencias (filosofía, sociología, psicología, política, etc.) que, a su vez, se nutre, en un proceso reflexivo, de la propia práctica educativa - que es siempre una práctica interactiva-, y del diálogo de esta con las diversas disciplinas que intervienen en el quehacer educativo. Ese cuerpo de conocimientos lo podemos tematizar como un campo disciplinar y profesional, como una teoría práctica. Esta perspectiva considera al maestro como un investigador que ejerce su trabajo de modo crítico y autónomo, es decir, como un auténtico pedagogo, lo que requiere una formación praxeológica que le permita el surgimiento y desarrollo de procesos de reflexión en y sobre la práctica. Definimos entonces la pedagogía como teoría de la acción educativa, en el sentido de una disciplina praxeológica (págs. 57-58).

En suma, la pedagogía es una teoría práctica, al tiempo reflexiva $\mathrm{y}$ prospectiva (y no tanto prescriptiva), $y, \quad$ por ende, 
orientada hacia el utópico futuro y no al quehacer presente.

\section{La pedagogía como teoría prác- tica: una definición esclarecedora}

La pedagogía sería, según esta concepción, una teoría, fundada en la experiencia educativa de cada maestro y orientada a la acción, cuya función es transformar la propia práctica formativa. El autor de tal "teoría práctica" sería un maestro profesional (un práctico) que se convierte en un teórico reflexivo de su acción con el fin de mejorarla. Eventualmente, puede ofrecer (socializar) su teoría a los demás. Insisto, desde Houssaye (1993), en este punto: en pedagogía es la misma persona quien practica y teoriza. De ahí su afirmación sobre la complejidad de ese monstruo conceptual que es el término "teoría práctica" aplicado a la pedagogía: "el envolvimiento mutuo y dialectico de la teoría y de la práctica educativa por la misma persona, sobre la misma persona. El pedagogo es un práctico-teórico de la acción educativa” (pág. 13).

Ahora bien, creo que la idea del maestro como profesional reflexivo e investigador de su propia práctica no ha terminado de concretarse, aunque sí se ha ido perfeccionando a lo largo de la historia, por la insistencia en la relación que existe entre el oficio de maestro y la reflexión/investigación como actitud y competencia profesional.

\section{La pregunta del pedagogo parte de su propia experiencia: ¿cómo hacer (lo) mejor?}

La respuesta que el maestro daría a su pregunta retorna praxeológicamente a su propia acción educativa. Cuando la presenta a otros su teoría lo hace bajo la forma de un discurso, que contiene una narrativa de una experiencia articuladora de tres elementos, tan mezclados entre sí que es difícil distinguirlos: (a) sus convicciones (valores), vinculadas a los propósitos que se tienen como maestro; (b) sus concepciones (teorías, modelos, saberes de referencia); y (c) sus acciones (prácticas que se asumen e implementan), conformando el triángulo pedagógico de todo maestro $^{1}$ (Esquema 1).

Las teorías prácticas típicas, en el campo educativo, son como aquellas de Pestalozzi, Freinet, Montessori, Freire y ciertos movimientos pedagógicos. Podemos extrapolar y pensar que muchos profesionales de la educación (maestros y directivos, pero también padres de familia) ejercen como pedagogos que generalmente desarrollan su teoría práctica sin hablar de ello, sin escribirlo ni transmitirlo. Así se pierden por millares las pedagogías porque tenemos dificultades para socializar, entre

\footnotetext{
${ }^{1}$ Esta idea puede completarse con lo que planteo en el capítulo 2 de mi libro $L a$ cuestión del método en pedagogía praxeológica (2017, págs. 55-92).
} 
nosotros, nuestra investigación y nuestras creaciones. $\mathrm{Y}$, por lo tanto, hacerlas (re)conocer.

El pedagogo (maestro): un teórico de su práctica

La pregunta del maestro, como investigador pedagógico (¿cómo hacerlo mejor?), la mayoría de las veces surge cuando hay un problema en el quehacer educativo cotidiano. Un bloqueo personal o colectivo, cuando se trabaja al interior de una corriente pedagógica.

A veces este ¿cómo hacerlo mejor? surge cuando brota una idea en nuestra cabeza: "¡Nunca más haré(mos) eso!”. Pero la pregunta inicial solo provocará un proceso investigativo cuando se convierta en ¿cómo puedo mejorar, con lo que sé (mis teorías, mis valores y mi práctica) y mis posibilidades de crear? Cuando reconocemos, planteamos y construimos mejor la problemática hallada porque nos atrevemos a preguntar ¿qué debería cambiar? o ¿qué debemos buscar o inventar?

Una reflexión que conducirá al pedagogo a mejorar el tejido de su teoría práctica personal, para lograr, después de tal o cual cambio, una nueva consistencia describible y argumentable, y tal vez, más adelante, nuevamente problematizable, para así dar lo mejor en el oficio de maestro. Porque "la verdadera palabra del pedagogo es la praxis, porque los hombres actúan en el mundo para humanizarlo, transformarlo y liberarlo. Y la educación tiene que ayudarlos a cumplir dicha tarea" (Juliao 2014, pág. 142).

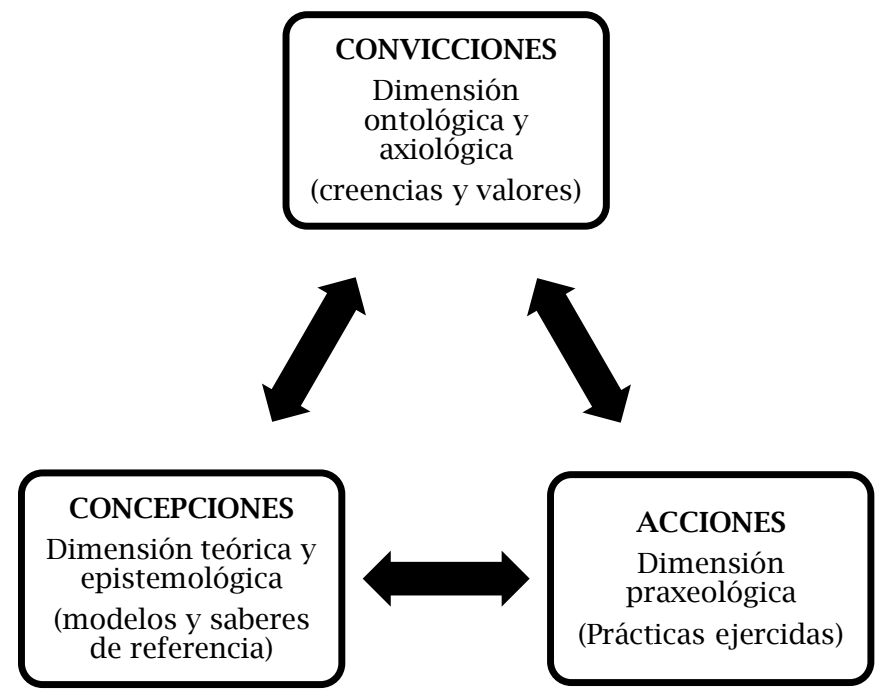

Esquema 1. Elementos de la teoría práctica del pedagogo/a (Construcción propia) 
Insisto en las imperfecciones de la pedagogía, pues le son intrínsecas

Esa consistencia buscada nunca será perfecta, pero la imperfección de la respuesta del pedagogo es su mayor riqueza: su investigación y su creatividad nacen y renacen de sus sucesivos intentos por reducir la brecha que separa sus teorías, sus prácticas, sus valores, de acuerdo con los problemas, las pruebas y las tensiones que halla en sus acciones educativas cotidianas. Las discrepancias entre la teoría y la práctica, las divergencias de perspectivas ideológicas, los debates y oposiciones entre actores de disímiles enfoques pedagógicos, pueden generar confusión y ser percibidos como fuentes de incoherencia.

En últimas, se trata de sortear la incoherencia entre el modelo educativo y las prácticas que lo concretan, así como de los procesos evaluativos de estas, poniéndolos al servicio del quehacer educativo. Sin embargo, el auténtico pedagogo/a renueva su teoría práctica en estas situaciones y, al hacerlo, (re)inventa los saberes pedagógicos que nacen de una reflexión sistemática y una voluntad de búsqueda. Pero el maestro también puede teorizar esos descubrimientos o innovaciones que surgen más aleatoriamente, como la casualidad (encontrar lo que no buscábamos) o la adaptación (aplicar modelos foráneos a nuestros contextos).
Así pues, el discurso del pedagogo parece fuera de sintonía con su actuación: el pedagogo no siempre dice lo que hace y a veces dice lo que no hace; a veces también lo hace mejor de lo que dice. Pero esta relación imperfecta entre decir $y$ hacer no es un defecto, aunque sí hay que mirar dentro de sí mismo para descubrir las propias resistencias:

Dicho de otra forma, pedagogo es aquel que educando a otros se pregunta cómo fue él educado, qué dolores y qué resistencia producían en su ser las incesantes intenciones de la educación. El ejercicio de la memoria se convierte en el factor determinante en la formación de un sujeto. Volver sobre sí y, a través de ello, descubrir la pregunta fundamental, constituye la esencia de la formación. De ahí que la experiencia y la capacidad se conviertan en el territorio de la formación y el tiempo en su principio de infinito. (Juliao, 2014, pág. 207)

\section{Un discurso que todavía hay que construir}

Cuando el trabajo no tiene éxito, ¿qué debo cambiar a mi práctica? Hameline (2002) lo expresa así: "En un momento en que se socava al pedagogo... ¿cómo seguir siendo pedagogo?" Si la envoltura mutua entre teoría y práctica siempre ha revelado relaciones complejas entre el decir $y$ el hacer pedagógicos, la 
explicación de nuestra pregunta ¿cómo hacer (lo) mejor? se presenta en discursos ya no tan dominadores como los de ayer. El he aquí como lo hice, luego así es que hay que hacerlo de ayer se convirtió en así es como propongo (o proponemos) hacerlo mejor. Porque sabemos que la forma de escribir y decir la pedagogía está en permanente cambio.

Los maestros ya no sienten la necesidad de asustar para resaltar esa singularidad de su quehacer reflexivo: sus propias teorías prácticas en eterna evolución y el nuevo saber pedagógico que siempre están construyendo. Esta nueva postura del pedagogo da más seriedad a la investigación educativa, pero, paradójicamente, debilita su reputación ante la sociedad. Si ayer nos burlábamos de la fe de carbonero de los pedagogos en su quehacer educativo, hoy escuchamos reproches como estos: "El pedagogo ya no lucha como antes" o " ¡Los maestros cambian y cambian sus propuestas de acción!". Lo anterior es una prueba de que la búsqueda pedagógica aún no se entiende como una mejora deseada y construida permanentemente para actuar mejor.

¿Cómo definir el saber
transferible que solo los
maestros producen?

La teoría práctica desarrollada por un maestro incluye, en el polo de su conocimiento, saberes de referencia múltiples, como los de las ciencias humanas y sociales, y en particular de las ciencias educativas. Sin embargo, estos, incluso si son numerosos, nunca bastan para actuar pedagógicamente bien. Por consiguiente, el pedagogo genera un saber nuevo y único, que satisface las necesidades de su acción, y de fundarla y explicarla coherente y pertinentemente.

Nacidos de la confrontación con el obstáculo y marcados por la experiencia, los saberes pedagógicos existen, siendo a la vez: (a) pragmáticos, pues se trata de "factibles" diversos (métodos, procesos, dispositivos); críticos, al sugerir la renuncia a prácticas anteriores; (c) políticos, pues dibujan alternativas; y (d) hermenéutico, dado que explican sus desafíos. Estos saberes hoy en día tienen dificultades para ser reconocidos como conocimiento transmisible porque aún tenemos problemas para reconocerlos, cuando es claro que no nacieron de un proceso de investigación científica y su validación no depende de procedimientos de control objetivables, pues los saberes de la pedagogía y las teorías prácticas que los incluyen deben aprobarse, tanto como probarse. Solo pueden probarse probándose a sí mismos.

Hay que aceptar que las ciencias no tienen la exclusividad del conocimiento, pues entre lo racional y lo irracional hay espacio para lo razonable: una razón práctica que no puede reducirse 
solo a la razón científica. Si bien no todos escribimos como los grandes pedagogos de la historia, muchos de nosotros sí somos pedagogos. Al menos en potencia. ¿Acaso no tratamos siempre de hacer coherente el tejido de nuestro pequeño triángulo pedagógico, al menos para nosotros mismos?

Concluyendo provisoriamente lo que no se puede concluir del todo

Para comprender bien lo aquí planteado, se requiere transformar la razón epistémica cimentada a lo largo de la historia e incorporar los nuevos saberes que hoy van surgiendo: muchos de ellos fruto de las teorías prácticas de los maestros. Eso significa admitir que:

a. Toda praxis educativa incluye una teoría, normalmente implícita, que la fundamenta.

b. Cada maestro tendrá que desentrañar su teoría implícita, para revelarla, objetivarla, comprenderla, transformarla y transferirla si es el caso.

c. Las prácticas educativas podrán ser mejoradas y transformadas desde la comprensión de las teorías implícitas (presupuestos, valores, propósitos) que las sostienen y de las condiciones históricas y contextuales de su desarrollo.

d. La práctica, como acción sociohistórica e intencional, está en permanente proceso de reconfiguración. Es decir, hay que asumirla con un compromiso social crítico.

e. Corresponde a la pedagogía, como teoría práctica, ser la interlocutora hermenéutica de las teorías implícitas en la praxis y mediar su posible transformación con fines emancipatorios.

f. También corresponde a la pedagogía ser la disciplina que convierta el sentido común pedagógico (el arte intuitivo presente en la praxis) en actos sistemáticos, desde los valores educativos, socialmente pertinentes, para una comunidad concreta.

La pedagogía, comprendida así, supera la histórica dualidad entre ser arte o ciencia educativa, para convertirse en aquella disciplina que transforma el arte educativo (el saber-hacer práctico intuitivo) en acción educativa razonada, proyectada e intencional. Ello significa que asumimos que la práctica educativa contiene acciones artesanales, espontáneas, intuitivas y creativas, que se van convirtiendo, praxeológicamente, en acciones reflexivas, afirmadas en teorías, fundadas mediante críticas, autocríticas y perspectivas de desempeño. Así, en el quehacer educativo, convive lo artístico con lo científico, expresado mediante la dinámica existente entre el ser y el hacer; entre el pensar y el realizar; entre el poder y el querer realizar. 


\section{Referencias}

De los Ríos, A. (2011). “Corrientes pedagógicas, tradiciones pedagógicas y Educación Especial: pensando históricamente la Educación Especial en América Latina". Revista RUEDES. Red universitaria de Educación Especial. 1(1), 3-21. http://bibliotecadigital.udea.ed u.co/handle/10495/3925

Fabre, M. (2002). "Existe-t-il des savoirs pédagogiques?" en J. H. Houssaye, M. Soëtard, D. Hameline y M. Fabre. Manifeste pour les pédagogues. Paris: ESF, pp. 99-124.

Flores-Ochoa, R. (1994). Hacia una pedagogía del conocimiento. Bogotá: McGraw Hill.

Houssaye, J. (dir.) (1993). La pédagogie: une encyclopédie pour aujourd'hui. Paris: ESF.

Juliao, C.G. (2014). Una pedagogía praxeológica. Bogotá: Uniminuto.

Juliao, C.G. (2017). La cuestión del método en pedagogía praxeológica. Bogotá: Uniminuto. 